\title{
Precision QCD Event Shapes at Hadron Colliders: The Transverse Energy-Energy Correlator in the Back-to-Back Limit
}

\author{
AnJie Gao, ${ }^{1, *}$ Hai Tao $\mathrm{Li}^{2,{ }^{2 \dagger}}$ Ian Moult, ${ }^{3,4, \$}$ and Hua Xing $\mathrm{Zhu}^{1, \S}$ \\ ${ }^{1}$ Zhejiang Institute of Modern Physics, Department of Physics, Zhejiang University, Hangzhou, 310027, China \\ ${ }^{2}$ Theoretical Division, MS B283, Los Alamos National Laboratory, Los Alamos, New Mexico 87545, USA \\ ${ }^{3}$ Berkeley Center for Theoretical Physics, University of California, Berkeley, California 94720, USA \\ ${ }^{4}$ Theoretical Physics Group, Lawrence Berkeley National Laboratory, Berkeley, California 94720, USA
}

(Received 22 January 2019; published 5 August 2019; corrected 9 August 2019)

\begin{abstract}
We present an operator-based factorization formula for the transverse energy-energy correlator (TEEC) hadron collider event shape in the back-to-back (dijet) limit. This factorization formula exhibits a remarkably symmetric form, being a projection onto a scattering plane of a more standard transverse momentum dependent factorization. Soft radiation is incorporated through a dijet soft function, which can be elegantly obtained to next-to-next-to-leading order (NNLO) due to the symmetries of the problem. We present numerical results for the TEEC resummed to next-to-next-to-leading logarithm (NNLL) matched to fixed order at the LHC. Our results constitute the first NNLL resummation for a dijet event shape observable at a hadron collider, and the first analytic result for a hadron collider dijet soft function at NNLO. We anticipate that the theoretical simplicity of the TEEC observable will make it indispensable for precision studies of QCD at the LHC, and as a playground for theoretical studies of factorization and its violation.
\end{abstract}

DOI: 10.1103/PhysRevLett.123.062001

Introduction.-Event shape observables, which measure the flow of radiation in a scattering event, play a central role in QCD. They allow for precision measurements of QCD parameters, such as the strong coupling constant, $\alpha_{s}$, as well as for probes of more subtle features of QCD, such as color evolution or factorization violation. While event shape observables in $e^{+} e^{-}$collisions are by now quite well understood, with calculations incorporating next-tonext-to leading order (NNLO) fixed order corrections [1-4], and next-to-next-to-next-to leading logarithmic $\left(\mathrm{N}^{3} \mathrm{LL}\right)$ resummation [5-8], the same level of understanding has not been achieved for event shape observables at hadron colliders. This is due both to the technical complexity of fixed order calculations with multiple legs, and to the failure of standard factorization formulas in the hadron collider context. The theoretical and experimental study of event shape observables at hadron colliders therefore provides genuinely new opportunities for improving our understanding of QCD.

An important aspect in the description of event shapes is the resummation of singular terms in kinematic limits. For hadron collider event shapes, NNLL resummation has been achieved for zero-jet [9-11] and one-jet event shapes [12].

Published by the American Physical Society under the terms of the Creative Commons Attribution 4.0 International license. Further distribution of this work must maintain attribution to the author(s) and the published article's title, journal citation, and DOI. Funded by SCOAP.
However, many interesting effects, namely nontrivial color evolution and amplitude level factorization violation, first occur for dijet event shapes, for which complete results are only available at NLL [13-18].

A number of recent developments, namely the calculation of the three loop soft anomalous dimension $[19,20]$, progress towards three jet production at NNLO [21-30], the illustration of the noncancellation of Glauber effects in dijet processes [31-33], the elucidation of amplitude-level factorization violation [34-36], and a formalism for the incorporation of factorization violation in the soft collinear effective theory (SCET) [37], motivate a renewed interest in the theoretical study of dijet event shapes.

In this Letter, we will study the transverse energyenergy-correlator (TEEC) observable [38,39],

$$
\frac{d \sigma}{d \cos \phi}=\sum_{a, b} \int d \sigma_{p p \rightarrow a+b+X} \frac{2 E_{T, a} E_{T, b}}{\left|\sum_{i} E_{T, i}\right|^{2}} \delta\left(\cos \phi_{a b}-\cos \phi\right),
$$

where the sum is over all pairs of hadrons, $E_{T}$ is the transverse energy of the hadrons, and $\phi_{a b}$ the azimuthal angle between the hadrons, as illustrated in Fig. 1. We note that due to the energy weighting in the definition of the observable, Eq. (1) is not truly a differential cross section. However, we follow the standard abuse of notation and denote it as such. For recent measurements of the TEEC for jets, see Refs. [40,41]. Building on significant recent 


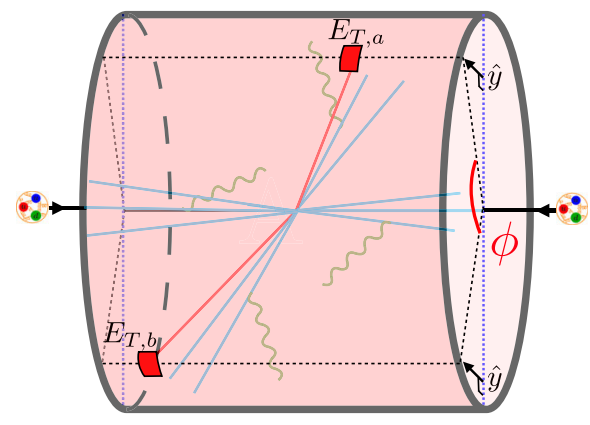

FIG. 1. The TEEC measures the $E_{T}$ weighted angular correlation of pairs of particles as a function of the angle $\phi$ in the transverse plane. In the $\phi \rightarrow \pi$ limit, it measures the momentum in the direction $\hat{y}$ perpendicular to the scattering plane spanned by the beam and jet axes, outlined in dashed blue.

progress in the understanding of the energy-energy corelator (EEC) observable [42-48], we will show that the TEEC exhibits a remarkable perturbative simplicity in the dijet limit, allowing for significant progress to be made in the understanding of hadron collider event shapes.

Factorization formula.-One of the main results of this Letter is an operator based factorization formula, derived in SCET [49-52], describing the singular behavior of the TEEC observable in the $\phi \rightarrow \pi$, or more conveniently, the $\tau \equiv \sin ^{2}[(\pi-\phi) / 2] \rightarrow 0$ limit. In this limit, the singular behavior of the observable is described by a dijet configuration, with collinear radiation along the beam and jet axes, as well as low energy soft radiation. The $\tau \rightarrow 0$ limit defines a scattering plane spanned by the beam axis and the axis of the outgoing jets (more precisely the transverse thrust axis). Collinear splittings and soft emissions recoil the particles correlated by the TEEC observable slightly from this plane, see Fig. 1. This singular region is similar to those considered for studying azimuthal correlations in DIS [53] or back-to-back jets in $e^{+} e^{-}$[54].

The simplicity of the TEEC lies in a relation between the azimuthal angle $\phi$ and the momentum perpendicular to the scattering plane, which we will denote as the $y$ component, as illustrated in Fig. 1. Consider two final state particles $k_{3}$ and $k_{4}$, whose transverse energy correlation is to be measured. In addition to the transverse momentum off the scattering plane due to final-state collinear splittings, they obtain transverse momentum from the recoil of the total soft momentum $k_{s, y}$, and from the momenta $k_{1, y}$ and $k_{2, y}$ of the incoming particles which enter the hard scattering. In the $\tau \rightarrow 0$ limit, we have the relation

$$
\tau=\frac{\left(\frac{k_{3, y}}{\xi_{3}}+\frac{k_{4, y}}{\xi_{4}}+k_{1, y}+k_{2, y}-k_{s, y}\right)^{2}}{4 P_{T}^{2}}+, \ldots,
$$

where $\xi_{3}$ and $\xi_{4}$ are the respective longitudinal momentum fractions of the two measured final state particles relative to the two leading jet momentum $p_{3}$ and $p_{4}$, and $P_{T}$ is the transverse momentum of $p_{3}$ and $p_{4}$ relative to the beam axis.

The relationship in Eq. (2) allows us to derive a factorization formula for the TEEC in the dijet limit in terms of standard transverse momentum dependent (TMD) beam and jet functions

$$
\begin{aligned}
\frac{d \sigma^{(0)}}{d \tau}= & \frac{1}{16 \pi s^{2}\left(1+\delta_{f_{3} f_{4}}\right) \sqrt{\tau}} \sum_{\text {channels }} \frac{1}{N_{\text {init }}} \int \frac{d y_{3} d y_{4} p_{T} d p_{T}^{2}}{\xi_{1} \xi_{2}} \int_{-\infty}^{\infty} \frac{d b}{2 \pi} e^{-2 i b \sqrt{\tau} p_{T} \operatorname{tr}\left[\mathbf{H}^{f_{1} f_{2} \rightarrow f_{3} f_{4}}\left(p_{T}, y^{*}, \mu\right) \mathbf{S}\left(b, y^{*}, \mu, \nu\right)\right]} \\
& \cdot B_{f_{1} / N_{1}}\left(b, \xi_{1}, \mu, \nu\right) B_{f_{2} / N_{2}}\left(b, \xi_{2}, \mu, \nu\right) J_{f_{3}}(b, \mu, \nu) J_{f_{4}}(b, \mu, \nu) .
\end{aligned}
$$

Here the superscript (0) indicates that this formula describes all contributions to the cross section that scale like $1 / \tau$ modulo logarithms, up to potentially factorization violating terms which occur first at $\mathrm{N}^{4} \mathrm{LO}$, and will be discussed shortly. This factorization formula is a sum over different $2 \rightarrow 2$ partonic scattering channels $f_{1}\left(p_{1}\right)$ $f_{2}\left(p_{2}\right) \rightarrow f_{3}\left(p_{3}\right) f_{4}\left(p_{4}\right)$, where $N_{\text {init }}$ is the corresponding spin- and color-averaged factor for each channel, $\sqrt{s}$ is the center-of-mass energy, $y_{3}, y_{4}$, and $p_{T}$ are the rapidity and transverse momentum of the two leading partonic jets at the lowest order in perturbation theory, and $\xi_{1}=p_{T}\left(e^{y_{3}}+\right.$ $\left.e^{y_{4}}\right) / \sqrt{s}$ and $\xi_{2}=p_{T}\left(e^{-y_{3}}+e^{-y_{4}}\right) / \sqrt{s}$ are the born-level initial-state momentum fractions. The dependence on the scattering channel is incorporated through the hard function $\mathbf{H}^{f_{1} f_{2} \rightarrow f_{3} f_{4}}\left(p_{T}, y^{*}, \mu\right)$, which depends on the $p_{T}$ and the single jet rapidity $y^{*}=\left(y_{3}-y_{4}\right) / 2$ in the partonic centerof-mass frame. Each of the functions in Eq. (3) depends on a virtuality renormalization scale $\mu$, and a rapidity renormalization scale $\nu[55,56]$. The associated renormalization group (RG) equations allow for the resummation of logarithms of $\tau$.

The soft and collinear dynamics in the dijet limit are described by beam functions, $B$, jet functions, $J$, and a soft function, $S$. The beam functions and jet functions in Eq. (3) are identical to the well-known TMD beam functions and EEC jet functions [47] (which are in turn related to the TMD fragmentation functions [57-59]). Therefore, the TEEC in the dijet limit provides a probe into both beam and jet TMD dynamics that is interesting to a broad community. Since the TMD beam and jet functions are standard objects, we do not discuss them further, but collect all the anomalous dimensions and matching coefficients in the Supplemental Material [60]. The TEEC soft function is new and will be discussed shortly. 
The factorization formula in Eq. (3) is expected to be violated at $\mathrm{N}^{4} \mathrm{LO}$ by Glauber gluons [88] which couple the different beam and jet functions. While the cancellation of Glauber gluons was shown for color singlet transverse moment distributions in the seminal works of Refs. [54,8893], it is expected that factorization should not hold for a dijet event shape [31,32,34-37,94-104]. Glauber contributions can potentially be incorporated in our formalism using Ref. [37], and indeed one of our primary motivations is to understand such violations by identifying a dijet observable with the simplest perturbative structure. Apart from a brief comment on the anomalous dimension of the soft function at $\mathrm{N}^{3} \mathrm{LO}$, we leave the study of violations of this factorization formula to future work, and restrict ourselves to NNLL accuracy where Eq. (3) holds.

Soft function.-The most complicated obstacle for precision calculations of multijet event shapes is the soft function, due to its dependence on multiple directions. (For recent progress towards numerical calculations of soft functions at NNLO, see Refs. [105-107].) A key feature of the TEEC which makes it particularly amenable to analytic higher order calculations is the simplicity of its soft function, which is defined as a vacuum expectation of Wilson lines,

$$
\mathbf{S}\left(b, y^{*}\right)=\left\langle 0\left|T\left[\mathbf{O}_{n_{1} n_{2} n_{3} n_{4}}\left(0^{\mu}\right)\right] \bar{T}\left[\mathbf{O}_{n_{1} n_{2} n_{3} n_{4}}^{\dagger}\left(b^{\mu}\right)\right]\right| 0\right\rangle,
$$

as illustrated in Fig. 2 (There the temporal direction has necessarily been suppressed). Here $\mathbf{O}_{n_{1} n_{2} n_{3} n_{4}}(x)=$ $\mathbf{Y}_{n_{1}} \mathbf{Y}_{n_{2}} \mathbf{Y}_{n_{3}} \mathbf{Y}_{n_{4}}(x)$, with $\mathbf{Y}_{n_{i}}(x)=\exp \left[i \int d s n_{i} \cdot A\left(s n_{i}+\right.\right.$ $x) \mathbf{T}_{i}$ ] a semi-infinite lightlike soft Wilson line, and $n_{i}^{\mu}=$ $p_{i}^{\mu} / p_{i}^{0}$ the lightlike direction of the incoming or outgoing parton in the partonic center-of-mass frame. The directions of the Wilson lines are standard and hence suppressed, as are gauge links at infinity. We have chosen coordinates such that $b^{\mu}=(0,0, b, 0)$ is in the direction $\hat{y}$ perpendicular to the scattering plane, $\hat{y} \cdot n_{i}=0$.

The soft function defined in Eq. (4) suffers from UV and rapidity divergences. Rapidity divergences are regulated

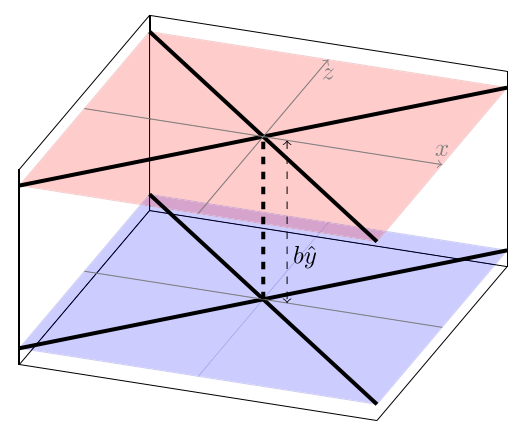

FIG. 2. The spatial structure of the TEEC soft function. Each set of Wilson lines lies in a scattering plane, and their relative displacement is perpendicular to these planes. using the exponential regulator of [108]. The soft function, which is a matrix in color space, satisfies the RG equation

$$
\frac{d \mathbf{S}}{d \ln \mu^{2}}=\frac{1}{2}\left(\boldsymbol{\Gamma}_{S}^{\dagger} \cdot \mathbf{S}+\mathbf{S} \cdot \boldsymbol{\Gamma}_{S}\right)
$$

with $[13,14,109,110]$

$\boldsymbol{\Gamma}_{S}=\sum_{i<j} \mathbf{T}_{i} \cdot \mathbf{T}_{j} \gamma_{\text {cusp }} \ln \frac{\nu^{2} n_{i} \cdot n_{j}}{2 \mu^{2}}-\sum_{i} \frac{c_{i}}{2} \gamma_{s} \mathbf{1}-\boldsymbol{\gamma}_{\text {quad }}$,

where $\nu$ is the rapidity scale, and $c_{i}=C_{F}$ or $C_{A}$ is the Casimir of the parton $i$. Here $\gamma_{\text {cusp }}$ is the cusp anomalous dimension [111], $\gamma_{s}$ is the threshold soft anomalous dimension [112] and $\boldsymbol{\gamma}_{\text {quad }}$ is the anomalous dimension for quadrupole color and kinematic entanglement, which first appears at three loops $[19,20]$. The evolution equation associated with the rapidity scale $\nu$ is

$$
\frac{d \mathbf{S}}{d \ln \nu^{2}}=\frac{1}{2}\left(\boldsymbol{\Gamma}_{y}^{\dagger} \cdot \mathbf{S}+\mathbf{S} \cdot \boldsymbol{\Gamma}_{y}\right)
$$

with

$$
\begin{aligned}
\boldsymbol{\Gamma}_{y}= & \left(\int_{\mu^{2}}^{b_{0}^{2} / b^{2}} \frac{d \bar{\mu}^{2}}{\bar{\mu}^{2}} \gamma_{\text {cusp }}\left[\alpha_{s}(\bar{\mu})\right]+\gamma_{r}\left[\alpha_{s}\left(b_{0} / b\right)\right]\right) \\
& \times \sum_{i} c_{i} \mathbf{1}+\boldsymbol{\gamma}_{X}\left[y^{*}, \alpha_{s}\left(b_{0} / b\right)\right] .
\end{aligned}
$$

This is the generalization of the rapidity RGE $[55,56]$ for color singlet production to dijet production at hadron colliders. Here $\gamma_{r}$ is the rapidity anomalous dimension for the color transverse momentum distribution [113], and $b_{0}=2 e^{-\gamma_{E}}$.

The color nondiagonal rapidity anomalous dimension, $\boldsymbol{\gamma}_{X}$, vanishes at one and two loops due to rescaling invariance, $n_{i} \rightarrow e^{\lambda_{i}} n_{i}$, which is sufficient for the NNLL resummation considered in this Letter. $\boldsymbol{\gamma}_{X}$ can potentially be nonzero at three loops where there is a scaling invariant cross ratio $n_{1} \cdot n_{3} n_{2} \cdot n_{4} /\left(n_{1} \cdot n_{2} n_{3} \cdot n_{4}\right)=\left(1-\tanh y^{*}\right)^{2} / 4$. The consistency of the factorization formula (derived from rapidity scale independence of the cross section) implies $\boldsymbol{\gamma}_{X}=0$ to all perturbative orders; however, since the factorization formula is expected to be violated, we do not take this as given. If $\boldsymbol{\gamma}_{X}=0$, it requires a symmetry explanation, and if not, it provides a direct window into factorization violation. Either way, we believe that the calculation of the TEEC soft function at three loops will provide considerable insight into rapidity factorization.

While the RG can be used to predict the logarithmic dependence of the soft function, its simple structure implies that the constants can also be easily computed. Writing its perturbative expansion as $\mathbf{S}=\sum\left(\alpha_{s} / 4 \pi\right)^{n} \mathbf{S}^{(n)}$, from a direct perturbative calculation, we find the beautiful relation 


$$
\begin{aligned}
\mathbf{S}^{(1)}\left(y^{*}, L_{b}, L_{\nu}\right)= & -\sum_{i<j}\left(\mathbf{T}_{i} \cdot \mathbf{T}_{j}\right) S_{\perp}^{(1)}\left(L_{b}, L_{\nu}+\ln \frac{n_{i} \cdot n_{j}}{2}\right), \\
\mathbf{S}^{(2)}\left(y^{*}, L_{b}, L_{\nu}\right)= & -\sum_{i<j}\left(\mathbf{T}_{i} \cdot \mathbf{T}_{j}\right) S_{\perp}^{(2)}\left(L_{b}, L_{\nu}+\ln \frac{n_{i} \cdot n_{j}}{2}\right), \\
& +\frac{1}{2 !}\left[\mathbf{S}^{(1)}\left(y^{*}, L_{b}, L_{\nu}\right)\right]^{2},
\end{aligned}
$$

where $S_{\perp}^{(n)}\left(L_{b}, L_{\nu}\right)$ is the $n$-loop TMD soft function for colorsinglet production at hadron colliders (which can be found up to three loops in Ref. [113]), and $L_{b}=\ln \left(\mu^{2} b^{2} / b_{0}^{2}\right)$, $L_{\nu}=\ln \left(\nu^{2} b^{2} / b_{0}^{2}\right)$. Details of this calculation will be presented in a future publication. Equation (9) is the first analytic result for a hadron collider dijet soft function at NNLO (The 2-jettiness soft function was computed numerically in Ref. [107]). The remarkable simplicity of the TEEC soft function should be compared with the soft functions for the $N$-jettiness observable [107,114-117], which already at one-loop, can only be computed numerically. We have also not included in Eq. (9) a purely imaginary component that does not contribute to the cross section, but is given in the Supplemental Material [60]. The reason for this simplicity is interesting, and deserves further comment. A soft function describes the expected value of radiation sourced by a configuration of Wilson lines, projected onto some direction(s). For the $N$-jettiness observable [118], these directions are the Wilson line directions themselves, which necessitates a partitioning of the phase space around the Wilson lines and leads to a complicated structure. For a dijet configuration, there is a unique direction perpendicular to the scattering plane defined by the four Wilson lines, which we have denoted $\hat{y}$, such that $\hat{y} \cdot n_{i}=0$ for all Wilson line directions $n_{i}$. This is the direction that is used to define the TEEC soft function, as shown in Fig. 2, and leads to its simplicity. In particular, it implies that the scale independent part of the TEEC soft function can only be function of scaling invariant cross ratio of $n_{i}$. This points to the TEEC soft function as the uniquely simple dijet soft function, and we believe this simplicity will facilitate further analytic studies.

Numerical results. -We can use our factorization formula in Eq. (3) to present numerical results for the LHC at $\sqrt{s}=13 \mathrm{TeV}$. We use the anti- $k_{T}$ algorithm [119] with cone size $R=0.4$ to select events with two leading jets having averaged jet $P_{T} \geq 250 \mathrm{GeV}$ and individual jet rapidity $|Y|<2.5$. The TEEC is computed for particles with rapidity $|y|<2.5$. Throughout, we will use the PDF4LHC15_NNLO_MC [120] parton distribution functions, and we take $\alpha_{s}\left(M_{Z}\right)=0.118$. An interesting feature of the TEEC is that due to the energy weighting, the rapidity cuts do not introduce so called nonglobal logarithms [121,122].

We begin by verifying that our factorization formula correctly reproduces the singular behavior as $\tau \rightarrow 0$ by comparing to the numerical code NLOJET++ $[123,124]$, which provides the LO and NLO QCD corrections to

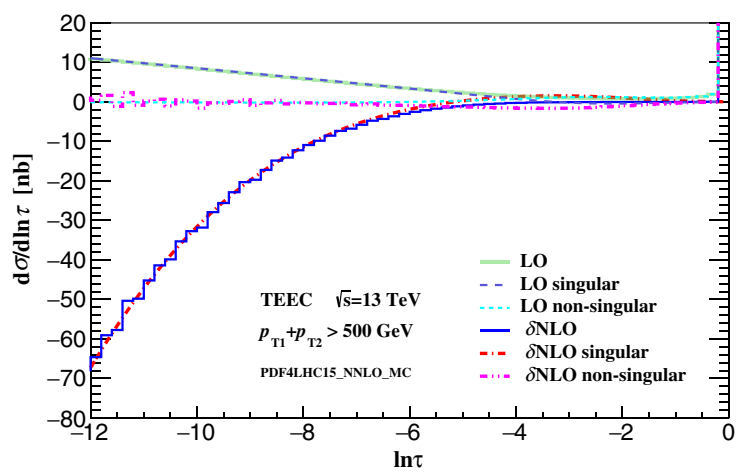

FIG. 3. The TEEC at LO and NLO in the dijet limit. Here $\delta$ NLO denotes only the NLO corrections.

three-jet production. We note that NLO QCD corrections to the TEEC for jets have been computed in Ref. [125] using NLOJET++, but here we are considering the TEEC for particles. Since the TEEC is first nonvanishing with a single emission from the dijet configuration, we use the perturbative counting for three-jet production for the matching. In Fig. 3 we show our factorization formula expanded to fixed order, compared with the numerical results of NLOJET++ for $\tau d \sigma / d \tau$, finding perfect agreement. This is highly nontrivial, as both calculations are rather involved, with nine different partonic channels at LO, and provides a strong check on the validity of our factorization formula. To the best of our knowledge, this is the first time that the singular behavior for a dijet differential distribution is under full control at this order.

In Fig. 4, we plot the full NLO prediction for $|d \sigma / d \phi|$ in the dijet limit, as well as its decomposition into the singular terms predicted by the factorization, and the nonsingular terms (power corrections) defined as the difference between the full fixed order calculation and the singular result. For $\phi \rightarrow 180^{\circ}$ the singular terms approach the full NLO predictions, as already demonstrated in Fig. 3, but here we can more clearly see the interplay between the singular and nonsingular terms. Since the TEEC effectively measures

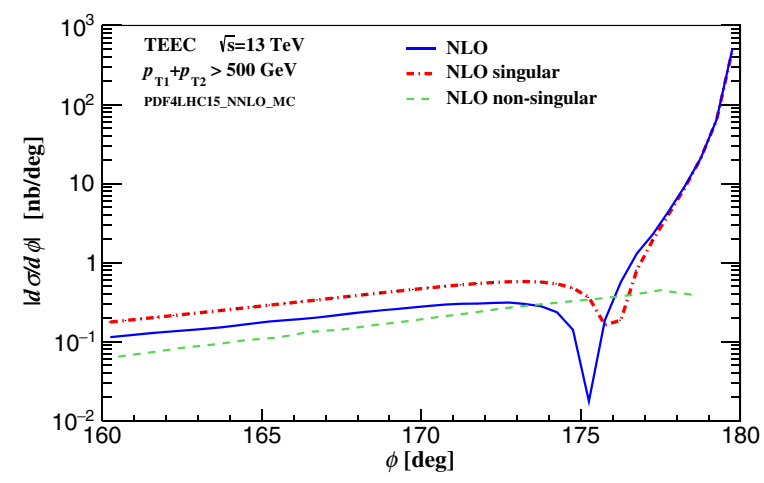

FIG. 4. Fixed order singular and nonsingular terms for the TEEC in the dijet limit. 


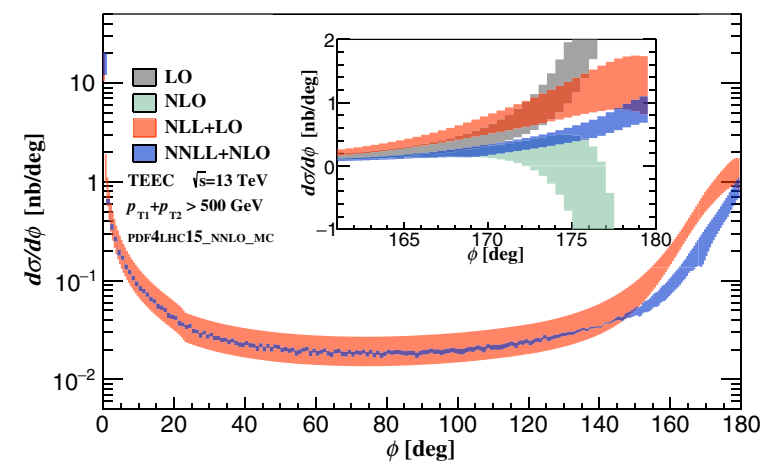

FIG. 5. The resummed TEEC distribution matched to fixed order at both NLL + LO and NNLL + NLO.

the $y$ component of an auxiliary transverse momentum $\left|q_{y}\right| \sim(\pi-\phi)$, this suggests that the power corrections start at $\mathcal{O}(\pi-\phi)$. It would be interesting to understand them further. Recent progress in the calculation of power corrections for transverse momentum type observables was made in Ref. [126].

In Fig. 5 we show resummed predictions for the TEEC at NLL and NNLL, matched to LO and NLO, respectively. Uncertainty bands are obtained by varying both the virtuality and rapidity scales up and down by a factor of two, see, e.g., Ref. [127]. As can be seen from Fig. 5, the resummation cures the divergences in the fixed order calculations as $\phi \rightarrow 180^{\circ}$, and it would be particularly interesting to have precise experimental measurements in this region. Also clear is the reduction of scale uncertainties from NLL + LO to NNLL + NLO, although we find that the perturbative corrections are large. We leave a detailed analysis of various uncertainties coming from scale variation, matching, and nonperturbative corrections to future work.

Conclusions. - In this Letter we have initiated the study of the TEEC hadron collider event shape. We have derived a factorization formula describing its singular behavior in the back-to-back (dijet) limit, and presented the first results for a dijet event shape at NNLL matched to NLO. The simplicity of the TEEC resides in its soft function, which we showed can be expressed in terms of a color singlet soft function through to NNLO.

There are a number of directions for further study and improvement. First, it will be interesting to compute the three-loop soft function for the TEEC to understand if $\boldsymbol{\gamma}_{X}$ is nonvanishing, and to understand the role of factorization violating terms at $\mathrm{N}^{3} \mathrm{LL}$. This will then enable matching to NNLO three-jet production once these become available [22-30]. The resummation of collinear logarithms at $\phi \rightarrow 0$ can be performed systematically using an extension of the jet calculus [128], and will be described in a forthcoming work. Finally, it would be interesting to compute the TEEC at strong coupling in planar $\mathcal{N}=4$ super Yang-Mills following Ref. [42], which could perhaps have relevance for heavy ion collisions. We believe the simplicity of the
TEEC observable provides a laboratory for precision studies of QCD at the LHC, and for studying the structure of factorization and factorization violation for hadron collider event shapes.

We thank Ben Nachman and Wei Wang for useful discussions, and Yu Jiao Zhu for checking part of the calculation. I. M. and H.X.Z. would like to express a special thanks to the Mainz Institute for Theoretical Physics (MITP) for its hospitality and support. A. J. G. and H. X. Z. are supported in part by the One Hundred Talent Program of Zhejiang University. H. T. L. is supported by the Los Alamos National Laboratory LDRD program. I. M. is supported by the Office of High Energy Physics of the U.S. DOE under Contract No. DE-AC02-05CH11231.

*gaoaj@zju.edu.cn

haitaoli@lanl.gov

Fianmoult@lbl.gov

§zhuhx@zju.edu.cn

[1] A. Gehrmann-De Ridder, T. Gehrmann, E. W. N. Glover, and G. Heinrich, J. High Energy Phys. 12 (2007) 094.

[2] A. Gehrmann-De Ridder, T. Gehrmann, E. W. N. Glover, and G. Heinrich, Phys. Rev. Lett. 99, 132002 (2007).

[3] S. Weinzierl, Phys. Rev. Lett. 101, 162001 (2008).

[4] S. Weinzierl, J. High Energy Phys. 06 (2009) 041.

[5] T. Becher and M. D. Schwartz, J. High Energy Phys. 07 (2008) 034.

[6] R. Abbate, M. Fickinger, A. H. Hoang, V. Mateu, and I. W. Stewart, Phys. Rev. D 83, 074021 (2011).

[7] Y.-T. Chien and M. D. Schwartz, J. High Energy Phys. 08 (2010) 058.

[8] A. H. Hoang, D. W. Kolodrubetz, V. Mateu, and I. W. Stewart, Phys. Rev. D 91, 094017 (2015).

[9] I. W. Stewart, F. J. Tackmann, and W. J. Waalewijn, Phys. Rev. Lett. 106, 032001 (2011).

[10] T. Becher, X. Garcia i Tormo, and J. Piclum, Phys. Rev. D 93, 054038 (2016); 93, 079905(E) (2016).

[11] T. Becher and X. Garcia i Tormo, J. High Energy Phys. 06 (2015) 071.

[12] T. T. Jouttenus, I. W. Stewart, F. J. Tackmann, and W. J. Waalewijn, Phys. Rev. D 88, 054031 (2013).

[13] N. Kidonakis, G. Oderda, and G. F. Sterman, Nucl. Phys. B525, 299 (1998).

[14] N. Kidonakis, G. Oderda, and G. F. Sterman, Nucl. Phys. B531, 365 (1998).

[15] A. Banfi, G. P. Salam, and G. Zanderighi, J. High Energy Phys. 08 (2004) 062.

[16] A. Banfi, G. P. Salam, and G. Zanderighi, J. High Energy Phys. 06 (2010) 038.

[17] P. Sun, C. P. Yuan, and F. Yuan, Phys. Rev. Lett. 113, 232001 (2014).

[18] A. Hornig, D. Kang, Y. Makris, and T. Mehen, J. High Energy Phys. 12 (2017) 043.

[19] Ø. Almelid, C. Duhr, and E. Gardi, Phys. Rev. Lett. 117, 172002 (2016).

[20] Ø. Almelid, C. Duhr, E. Gardi, A. McLeod, and C. D. White, J. High Energy Phys. 09 (2017) 073. 
[21] S. Badger, H. Frellesvig, and Y. Zhang, J. High Energy Phys. 12 (2013) 045.

[22] T. Gehrmann, J. M. Henn, and N. A. Lo Presti, Phys. Rev. Lett. 116, 062001 (2016); 116, 189903(E) (2016).

[23] D. C. Dunbar and W. B. Perkins, Phys. Rev. D 93, 085029 (2016).

[24] S. Abreu, F. Febres Cordero, H. Ita, B. Page, and M. Zeng, Phys. Rev. D 97, 116014 (2018).

[25] S. Badger, C. Brønnum-Hansen, H. B. Hartanto, and T. Peraro, Phys. Rev. Lett. 120, 092001 (2018).

[26] S. Badger, C. Brønnum-Hansen, H. B. Hartanto, and T. Peraro, J. High Energy Phys. 01 (2019) 186.

[27] S. Abreu, F. Febres Cordero, H. Ita, B. Page, and V. Sotnikov, J. High Energy Phys. 11 (2018) 116.

[28] S. Abreu, J. Dormans, F. Febres Cordero, H. Ita, and B. Page, Phys. Rev. Lett. 122, 082002 (2019).

[29] S. Abreu, L. J. Dixon, E. Herrmann, B. Page, and M. Zeng, Phys. Rev. Lett. 122, 121603 (2019).

[30] D. Chicherin, J. M. Henn, P. Wasser, T. Gehrmann, Y. Zhang, and S. Zoia, Phys. Rev. Lett. 122, 121602 (2019).

[31] J. Collins and J.-W. Qiu, Phys. Rev. D 75, 114014 (2007).

[32] J. Collins, arXiv:0708.4410.

[33] P. J. Mulders and T. C. Rogers, arXiv:1102.4569.

[34] S. Catani, D. de Florian, and G. Rodrigo, J. High Energy Phys. 07 (2012) 026.

[35] J. R. Forshaw, M. H. Seymour, and A. Siodmok, J. High Energy Phys. 11 (2012) 066.

[36] M. D. Schwartz, K. Yan, and H. X. Zhu, Phys. Rev. D 96, 056005 (2017).

[37] I. Z. Rothstein and I. W. Stewart, J. High Energy Phys. 08 (2016) 025.

[38] C. L. Basham, L. S. Brown, S. D. Ellis, and S. T. Love, Phys. Rev. Lett. 41, 1585 (1978).

[39] A. Ali, E. Pietarinen, and W. J. Stirling, Phys. Lett. 141B, 447 (1984).

[40] G. Aad et al. (ATLAS Collaboration), Phys. Lett. B 750, 427 (2015).

[41] M. Aaboud et al. (ATLAS Collaboration), Eur. Phys. J. C 77, 872 (2017).

[42] D. M. Hofman and J. Maldacena, J. High Energy Phys. 05 (2008) 012.

[43] A. V. Belitsky, S. Hohenegger, G. P. Korchemsky, E. Sokatchev, and A. Zhiboedov, Nucl. Phys. B884, 305 (2014).

[44] A. V. Belitsky, S. Hohenegger, G. P. Korchemsky, E. Sokatchev, and A. Zhiboedov, Nucl. Phys. B884, 206 (2014).

[45] A. V. Belitsky, S. Hohenegger, G. P. Korchemsky, E. Sokatchev, and A. Zhiboedov, Phys. Rev. Lett. 112, 071601 (2014).

[46] V. Gonçalves, J. High Energy Phys. 04 (2015) 150.

[47] I. Moult and H. X. Zhu, J. High Energy Phys. 08 (2018) 160.

[48] L. J. Dixon, M.-X. Luo, V. Shtabovenko, T.-Z. Yang, and H. X. Zhu, Phys. Rev. Lett. 120, 102001 (2018).

[49] C. W. Bauer, S. Fleming, and M. E. Luke, Phys. Rev. D 63, 014006 (2000).

[50] C. W. Bauer, S. Fleming, D. Pirjol, and I. W. Stewart, Phys. Rev. D 63, 114020 (2001).
[51] C. W. Bauer and I. W. Stewart, Phys. Lett. B 516, 134 (2001).

[52] C. W. Bauer, D. Pirjol, and I. W. Stewart, Phys. Rev. D 65, 054022 (2002).

[53] A. Banfi, G. Marchesini, and G. Smye, J. High Energy Phys. 04 (2002) 024.

[54] J. C. Collins and D. E. Soper, Nucl. Phys. B193, 381 (1981); B213, 545(E) (1983).

[55] J.-y. Chiu, A. Jain, D. Neill, and I. Z. Rothstein, Phys. Rev. Lett. 108, 151601 (2012).

[56] J.-Y. Chiu, A. Jain, D. Neill, and I. Z. Rothstein, J. High Energy Phys. 05 (2012) 084.

[57] J. Collins, Foundations of Perturbative QCD (Cambridge University Press, Cambridge, England, 2013).

[58] M. G. Echevarria, I. Scimemi, and A. Vladimirov, J. High Energy Phys. 09 (2016) 004.

[59] M.-x. Luo, X. Wang, X. Xu, L. L. Yang, T.-Z. Yang, and H. X. Zhu (to be published).

[60] See Supplemental Material at http://link.aps.org/ supplemental/10.1103/PhysRevLett.123.062001 for the relevant anomalous dimension and matching coefficients used for the results presented in the main article, which included Refs. [61-87].

[61] O. V. Tarasov, A. A. Vladimirov, and A. Y. Zharkov, Phys. Lett. 93B, 429 (1980).

[62] S. A. Larin and J. A. M. Vermaseren, Phys. Lett. B 303, 334 (1993).

[63] S. Moch, J. A. M. Vermaseren, and A. Vogt, Nucl. Phys. B688, 101 (2004).

[64] S. Moch, J. A. M. Vermaseren, and A. Vogt, J. High Energy Phys. 08 (2005) 049.

[65] S. Moch, J. A. M. Vermaseren, and A. Vogt, Phys. Lett. B 625, 245 (2005).

[66] A. Idilbi, X.-d. Ji, J.-P. Ma, and F. Yuan, Phys. Rev. D 73, 077501 (2006).

[67] A. Idilbi, X. dong Ji, and F. Yuan, Nucl. Phys. B753, 42 (2006).

[68] T. Becher, M. Neubert, and B. D. Pecjak, J. High Energy Phys. 01 (2007) 076.

[69] A. A. Vladimirov, Phys. Rev. Lett. 118, 062001 (2017).

[70] A. Vladimirov, J. High Energy Phys. 04 (2018) 045.

[71] I. Moult, I. W. Stewart, F. J. Tackmann, and W. J. Waalewijn, Phys. Rev. D 93, 094003 (2016).

[72] Z. Kunszt, A. Signer, and Z. Trocsanyi, Nucl. Phys. B411, 397 (1994).

[73] C. Anastasiou, E. N. Glover, C. Oleari, and M. TejedaYeomans, Nucl. Phys. B601, 318 (2001).

[74] C. Anastasiou, E. N. Glover, C. Oleari, and M. TejedaYeomans, Nucl. Phys. B601, 341 (2001).

[75] E. N. Glover, C. Oleari, and M. Tejeda-Yeomans, Nucl. Phys. B605, 467 (2001).

[76] Z. Bern, A. De Freitas, and L. J. Dixon, J. High Energy Phys. 03 (2002) 018.

[77] Z. Bern, A. De Freitas, and L. J. Dixon, J. High Energy Phys. 06 (2003) 028.

[78] E. W. N. Glover and M. E. Tejeda-Yeomans, J. High Energy Phys. 06 (2003) 033.

[79] E. W. N. Glover, J. High Energy Phys. 04 (2004) 021.

[80] A. De Freitas and Z. Bern, J. High Energy Phys. 09 (2004) 039. 
[81] R. Kelley and M. D. Schwartz, Phys. Rev. D 83, 045022 (2011).

[82] A. Broggio, A. Ferroglia, B. D. Pecjak, and Z. Zhang, J. High Energy Phys. 12 (2014) 005.

[83] T. Gehrmann, T. Lubbert, and L. L. Yang, Phys. Rev. Lett. 109, 242003 (2012).

[84] T. Gehrmann, T. Luebbert, and L. L. Yang, J. High Energy Phys. 06 (2014) 155.

[85] T. Lübbert, J. Oredsson, and M. Stahlhofen, J. High Energy Phys. 03 (2016) 168.

[86] J. G. M. Gatheral, Phys. Lett. 133B, 90 (1983).

[87] J. Frenkel and J. C. Taylor, Nucl. Phys. B246, 231 (1984).

[88] J. C. Collins, D. E. Soper, and G. F. Sterman, Nucl. Phys. B308, 833 (1988).

[89] J. C. Collins and D. E. Soper, Nucl. Phys. B197, 446 (1982).

[90] J.C. Collins and G. F. Sterman, Nucl. Phys. B185, 172 (1981).

[91] J. C. Collins, D. E. Soper, and G. F. Sterman, Nucl. Phys. B250, 199 (1985).

[92] J. C. Collins, D. E. Soper, and G. F. Sterman, Nucl. Phys. B261, 104 (1985).

[93] J. C. Collins, D. E. Soper, and G. F. Sterman, Adv. Ser. Dir. High Energy Phys. 5, 1 (1989).

[94] C. J. Bomhof, P. J. Mulders, W. Vogelsang, and F. Yuan, Phys. Rev. D 75, 074019 (2007).

[95] T. C. Rogers and P. J. Mulders, Phys. Rev. D 81, 094006 (2010).

[96] M. G. A. Buffing and P. J. Mulders, Phys. Rev. Lett. 112, 092002 (2014).

[97] J. R. Gaunt, J. High Energy Phys. 07 (2014) 110.

[98] M. Zeng, J. High Energy Phys. 10 (2015) 189.

[99] J. R. Forshaw, A. Kyrieleis, and M. H. Seymour, J. High Energy Phys. 09 (2008) 128.

[100] J. R. Forshaw, A. Kyrieleis, and M. H. Seymour, J. High Energy Phys. 08 (2006) 059.

[101] R. Ángeles Martínez, M. De Angelis, J. R. Forshaw, S. Plätzer, and M. H. Seymour, J. High Energy Phys. 05 (2018) 044.

[102] R. Ángeles Martínez, J. R. Forshaw, and M. H. Seymour, Phys. Rev. Lett. 116, 212003 (2016).

[103] R. Ángeles-Martínez, J. R. Forshaw, and M. H. Seymour, J. High Energy Phys. 12 (2015) 091.

[104] M. D. Schwartz, K. Yan, and H. X. Zhu, Phys. Rev. D 97, 096017 (2018).

[105] G. Bell, R. Rahn, and J. Talbert, Nucl. Phys. B936, 520 (2018).

[106] G. Bell, R. Rahn, and J. Talbert, arXiv:1812.08690.
[107] G. Bell, B. Dehnadi, T. Mohrmann, and R. Rahn, Proceedings, 14th DESY Workshop on Elementary Particle Physics: Loops and Legs in Quantum Field Theory 2018 (LL2018): St. Goar, Germany, 2018, Proc. Sci., LL2018 (2018) 044 [arXiv:1808.07427].

[108] Y. Li, D. Neill, and H. X. Zhu, arXiv:1604.00392 [Phys. Rev. D (to be published)].

[109] S. M. Aybat, L. J. Dixon, and G. F. Sterman, Phys. Rev. D 74, 074004 (2006).

[110] S. M. Aybat, L. J. Dixon, and G. F. Sterman, Phys. Rev. Lett. 97, 072001 (2006).

[111] G. Korchemsky and A. Radyushkin, Nucl. Phys. B283, 342 (1987).

[112] Y. Li, A. von Manteuffel, R. M. Schabinger, and H.X. Zhu, Phys. Rev. D 91, 036008 (2015).

[113] Y. Li and H. X. Zhu, Phys. Rev. Lett. 118, 022004 (2017).

[114] T. T. Jouttenus, I. W. Stewart, F. J. Tackmann, and W. J. Waalewijn, Phys. Rev. D 83, 114030 (2011).

[115] R. Boughezal, X. Liu, and F. Petriello, Phys. Rev. D 91, 094035 (2015).

[116] J. M. Campbell, R. K. Ellis, R. Mondini, and C. Williams, Eur. Phys. J. C 78, 234 (2018).

[117] H. T. Li and J. Wang, Phys. Lett. B 784, 397 (2018).

[118] I. W. Stewart, F. J. Tackmann, and W. J. Waalewijn, Phys. Rev. Lett. 105, 092002 (2010).

[119] M. Cacciari, G. P. Salam, and G. Soyez, J. High Energy Phys. 04 (2008) 063.

[120] J. Butterworth et al., J. Phys. G 43, 023001 (2016).

[121] M. Dasgupta and G. P. Salam, Phys. Lett. B 512, 323 (2001).

[122] A. Banfi, G. Marchesini, and G. Smye, J. High Energy Phys. 08 (2002) 006.

[123] Z. Nagy, Phys. Rev. Lett. 88, 122003 (2002).

[124] Z. Nagy, Phys. Rev. D 68, 094002 (2003).

[125] A. Ali, F. Barreiro, J. Llorente, and W. Wang, Phys. Rev. D 86, 114017 (2012).

[126] M. A. Ebert, I. Moult, I. W. Stewart, F. J. Tackmann, G. Vita, and H. X. Zhu, J. High Energy Phys. 04 (2019) 123.

[127] X. Chen, T. Gehrmann, E. W. N. Glover, A. Huss, Y. Li, D. Neill, M. Schulze, I. W. Stewart, and H. X. Zhu, Phys. Lett. B 788, 425 (2019).

[128] K. Konishi, A. Ukawa, and G. Veneziano, Nucl. Phys. B157, 45 (1979).

Correction: Email addresses for all authors were missing at publication and have been inserted as byline footnotes. 